\title{
MEK modulates force-fluctuation-induced relengthening of canine tracheal smooth muscle
}

\author{
M.L. Dowell**\#, T.L. Lavoie*, O.J. Lakser\#, N.O. Dulin*, J.J. Fredberg", \\ W.T. Gerthoffer ${ }^{+}$, C.Y. Seow ${ }^{\S}$, R.W. Mitchell*,f and J. Solway*,\#,f
}

ABSTRACT: Tidal breathing, and especially deep breathing, is known to antagonise bronchoconstriction caused by airway smooth muscle (ASM) contraction; however, this bronchoprotective effect of breathing is impaired in asthma. Force fluctuations applied to contracted ASM in vitro cause it to relengthen, force-fluctuation-induced relengthening (FFIR). Given that breathing generates similar force fluctuations in ASM, FFIR represents a likely mechanism by which breathing antagonises bronchoconstriction. Thus it is of considerable interest to understand what modulates FFIR, and how ASM might be manipulated to exploit this phenomenon. It was demonstrated previously that p38 mitogen-activated protein kinase (MAPK) signalling regulates FFIR in ASM strips. Here, it was hypothesised that the MAPK kinase (MEK) signalling pathway also modulates FFIR.

In order to test this hypothesis, changes in FFIR were measured in ASM treated with the MEK inhibitor, U0126 (1,4-diamino-2,3-dicyano-1,4-bis[2-aminophenylthio]butadiene).

Increasing concentrations of U0126 caused greater FFIR. U0126 reduced extracellular signalregulated kinase 1/2 phosphorylation without affecting isotonic shortening or 20-kDa myosin light chain and p38 MAPK phosphorylation. However, increasing concentrations of U0126 progressively blunted phosphorylation of high-molecular-weight caldesmon (h-caldesmon), a downstream target of MEK. Thus changes in FFIR exhibited significant negative correlation with $\mathrm{h}$ caldesmon phosphorylation.

The present data demonstrate that FFIR is regulated through MEK signalling, and suggest that the role of MEK is mediated, in part, through caldesmon.

KEYWORDS: Airway smooth muscle, asthma, bronchoconstriction, smooth muscle mechanics, tidal breathing

B ronchoconstriction and airflow obstruction during an asthma attack are, in part, a result of excessive contraction of airway smooth muscle (ASM). The extent of airway narrowing during bronchial provocation is not predicted solely, or even principally, by the ability of ASM to generate isometric force [1-10]. In animals and normal human individuals subjected to bronchial provocation, airway constriction is dramatically attenuated by tidal breathing [9, 11-13] and deep inspirations, which are amongst the most potent of all known bronchodilatory interventions $[10,14]$. These results probably arise because, during breathing, force fluctuations transmitted from lung parenchyma act directly on contracted ASM to influence its ability to maintain a shortened state; as a result, airway calibre equilibrates dynamically $[3,4,8]$ at a length longer than that in the static state.
In isolated bovine ASM that is contracted isotonically, initiation of force fluctuations causes appreciable muscle relengthening [4, 15]. It has been demonstrated that such force-fluctuationinduced relengthening (FFIR) is physiologically regulated $[15,16]$. For example, pharmacological inhibition of the activity of p38 mitogen-activated protein kinase (MAPK), a signalling kinase known to modulate smooth muscle contraction [17], also potentiates FFIR in bovine tracheal smooth muscle (TSM) strips [15]. Since MAPK kinase (MEK) has also been implicated in regulation of smooth muscle contraction [18-20], it was hypothesised that MEK signalling might also affect FFIR.

Acetylcholine (ACh) activation of G-proteincoupled muscarinic receptors [21] results in the initiation of several signalling cascades. Activation of myosin light chain (MLC) kinase and MEK

\section{AFFILIATIONS}

Depts of *Medicine and

\#Pediatrics, Section of Pulmonary and Critical Care Medicine, The University of Chicago, Chicago, IL, "Physiology Program, Harvard School of Public Health, Boston, MA ${ }^{+}$Dept of Biochemistry and Molecular Biology, University of South Alabama, Mobile, AL, USA, and ${ }^{\S}$ Dept of Pathology and Laboratory Medicine, University of British Columbia, Vancouver, BC, Canada, ${ }^{f}$ Both authors contributed equally to this study.

\section{CORRESPONDENCE}

M.L. Dowell

Section of Pulmonary Medicine, Dept of Pediatrics

The University of Chicago

5841 S. Maryland Avenue

MC4064

Chicago

IL 60637

USA

E-mail: mdowell@

peds.bsd.uchicago.edu

Received:

Oct 092009

Accepted after revision:

Jan 122010

First published online:

Jan 282010 
promotes 20-kDa MLC (MLC20) phosphorylation via elevated intracellular calcium levels and Ras signalling, respectively. Also the Ras/Raf/MEK signalling cascade activates (phosphorylates) extracellular signal-regulated kinase (ERK) 1/2 and its downstream regulators of contraction such as integrin-linked kinase [22, 23] and caldesmon [24-27].

In the present study, the hypothesis that the MEK signalling pathway regulates FFIR in TSM was tested using an inhibitor of MEK, U0126 (1,4-diamino-2,3-dicyano-1,4-bis[2-aminophenylthio]butadiene). It was found that, during MEK inhibition, changes in FFIR ( $\triangle$ FFIR) correlated inversely with caldesmon phosphorylation. In contrast, MEK inhibition reduced neither MLC20, p38 MAPK and 27-kDa heat shock protein (Hsp27) phosphorylation nor isotonic shortening. These results demonstrate that MEK is a key regulator of FFIR, and suggests that its effect is mediated, at least in part, through caldesmon phosphorylation.

\section{METHODS}

\section{Tissue preparation}

In accordance with Institutional Animal Care and Use Committee-approved protocols, dogs were anaesthetised and euthanised by an overdose of pentobarbital sodium $(30 \mathrm{mg} \cdot \mathrm{kg}$ body weight ${ }^{-1}$ intravenously). Tracheae were removed and rinsed in Krebs-Henseleit $(\mathrm{KH})$ solution containing $115 \mathrm{mM}$ $\mathrm{NaCl}, 25 \mathrm{mM} \mathrm{NaCO}, 1.38 \mathrm{mM} \mathrm{KH} \mathrm{KO}_{4}, 2.51 \mathrm{mM} \mathrm{KCl}$, $2.46 \mathrm{mM} \mathrm{MgCl}, 2.5 \mathrm{mM} \mathrm{CaCl} 2$ and $11.2 \mathrm{mM}$ dextrose. The $\mathrm{KH}$ solution was gassed with $95 \%$ oxygen $/ 5 \%$ carbon dioxide in order to maintain a $\mathrm{pH}$ of 7.3-7.5, and all studies were conducted at $37^{\circ} \mathrm{C}$ in $\mathrm{KH}$ solution. Some tissues were stored for up to 4 days at $4^{\circ} \mathrm{C}$ prior to study, without any apparent effect on the results.

As described previously [16], parallel fibred bundles of TSM measuring $0.25-0.50 \mathrm{~mm}$ in width and $0.5-1.0 \mathrm{~mm}$ in depth were dissected free of overlying connective tissue and epithelium. Each muscle strip was attached at either end in aluminium foil clips (Laser Services Inc., Westford, MA, USA) that held the muscle firmly. One clip was slipped over a rigidly held hook at one end of a horizontal dip-tray style of organ bath. The other clip was fastened to a hook connected to a 300B lever arm/force transducer (Aurora Scientific, Aurora, ON, Canada) that measured both force output and length changes, which were monitored using ADInstruments Powerlab Chart software (ADInstruments, Colorado Springs, CO, USA).

\section{Tissue equilibration}

Over $\sim 90 \mathrm{~min}$, tissues were contracted periodically ( $\sim 10-15$ min intervals) using $43 \mathrm{mM} \mathrm{KCl}$-substituted $\mathrm{KH}$ solution to establish the reference length (Lref). Lref ranged 3.5-8.0 mm. Resting length was adjusted between responses until developed force was maximal and repeatable. As previously shown, this concentration of $\mathrm{KCl}$ is optimal for canine TSM voltagedependent contraction and does not cause release of ACh from neural elements in the tissue preparation [28].

\section{Isometric protocols}

After equilibration, TSM strips were exposed to U0126 (Promega, Madison, WI, USA; 15 or $30 \mu \mathrm{M}$ ) or vehicle for $\geqslant 45 \mathrm{~min}$, and then cumulative ACh concentration-response studies were performed $\left(1 \times 10^{-9}-1 \times 10^{-4} \mathrm{M}\right)$. All isometric data in response to $\mathrm{ACh}$ were normalised to the contraction elicited during the last exposure of each muscle to $43 \mathrm{mM} \mathrm{KCl}-$ substituted $\mathrm{KH}$ solution.

\section{FFIR protocols}

After equilibration, tissues were isometrically contracted by switching the perfusion solution to $\mathrm{KH}$ solution containing $1 \times 10^{-4} \mathrm{M}$ Ach, and the maximal response ( Fmax) was noted. Muscles were allowed to relax by reperfusing with $\mathrm{KH}$ solution alone. Lref and Fmax were then used as the base parameters for the force oscillation protocols described below (fig. 1a). Tissues were re-exposed to $1 \times 10^{-4} \mathrm{M} \mathrm{ACh} 20 \mathrm{~min}$ after force had reached the relaxed baseline, and permitted to shorten isotonically against an afterload of 32\% Fmax for 20 min. During continued ACh exposure, sinusoidal force oscillations $(0.2 \mathrm{~Hz}$, with amplitude of $\pm 16 \%$ Fmax $)$ were then superimposed (to simulate tidal breathing) for $20 \mathrm{~min}[4,15$, 16, 29]. FFIR was noted at the end of $20 \mathrm{~min}$ and ACh was washed out. Muscles were oscillated using Aurora Scientific Dynamic Muscle Control software. Muscle force and length outputs were acquired through a National Instruments PCI6036E data acquisition board (National Instruments, Austin, TX, USA); data were monitored and collected using both LabView-based Dynamic Muscle Control (Aurora Scientific) and ADInstruments PowerLab Chart software.

Tissues were then incubated for $1 \mathrm{~h}$ in $\mathrm{KH}$ solution containing 3,15 or $30 \mu \mathrm{M}$ U0126 or $0.03 \%$ dimethylsulfoxide (DMSO; vehicle control). Prior studies had shown that this concentration of DMSO did not affect subsequent length changes in the isotonic/force oscillation protocol [16]. These concentrations of MEK inhibitor were chosen based on literature values and our own studies on a separate cohort of TSM strips that showed them to have only minimal effects on isometric force (see Isometric protocols section above). In addition, these concentrations did not affect isotonic shortening in response to $\mathrm{ACh}$, MLC20 phosphorylation or p38 MAPK activation state in the present study (see Results section). After this equilibration period, the isotonic contraction protocol was repeated in the continued presence of inhibitor or vehicle (fig. 1). Muscle length changes during contractions before and after inhibitor treatment were expressed as a percentage of Lref. Near the end of the second contraction sequence, but before force fluctuations ceased, most tissues were flash-frozen in liquid nitrogen. Strips were then transferred to dry-ice-chilled acetone (containing $5 \%$ trichloroacetic acid and $10 \mathrm{mM}$ dithiothreitol) and stored at $-80^{\circ} \mathrm{C}$ for protein extraction and western blot analysis.

\section{Jasplakinolide and U0126 combined studies}

Previously, it was shown that contractile actin polymerisation plays a key role in regulating FFIR [16]. This protocol was designed to test the hypothesis that MEK inhibition enhances FFIR by reducing actin filament stability. Tissues were equilibrated, Fmax determined and the initial isotonic/force oscillation protocol performed (as above). TSM strips were allowed to relax and then incubated for $1 \mathrm{~h}$ in jasplakinolide (EMD Biosciences, San Diego, CA, USA; $500 \mathrm{nM}$ ), an agent that stabilises actin filaments and promotes polymerisation. While still in the presence of jasplakinolide, the contraction sequence was repeated. After completion of this second contraction 

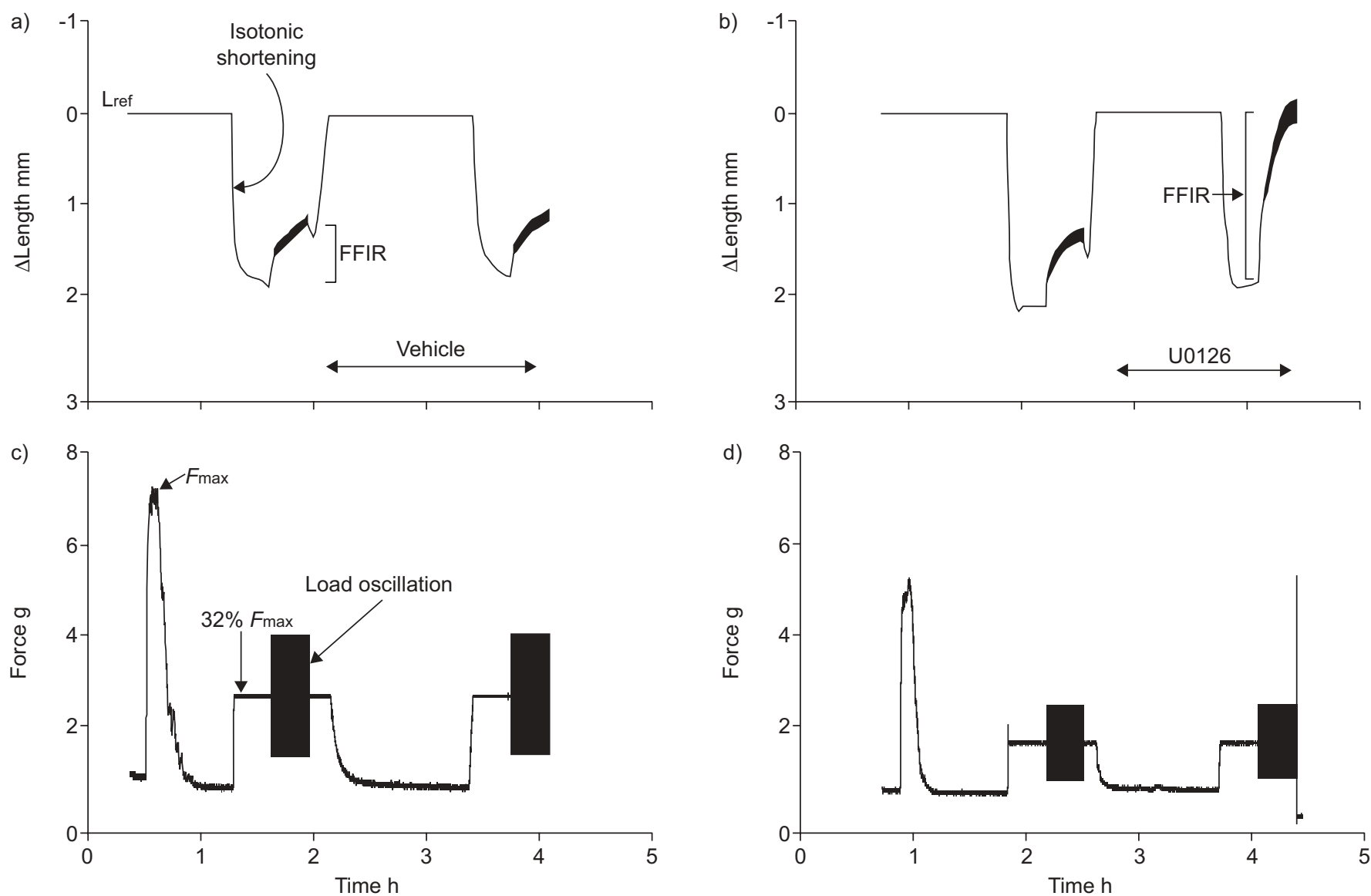

FIGURE 1. Experimental protocol. Tracheal smooth muscle strips were exposed to U0126 (1,4-diamino-2,3-dicyano-1,4-bis[2-aminophenylthio]butadiene) or vehicle (control) for $45 \mathrm{~min}$, after which the oscillation protocol was then repeated. The difference $(\Delta)$ in force-fluctuation-induced relengthening (FFIR) after versus before treatment was determined. Load oscillation was performed at $32 \pm 16 \%$ of the maximum force of contraction (Fmax). Near the end of the second contraction sequence, tissues were flash-frozen in liquid nitrogen For the vehicle-treated (control) muscle strip (a and c), there was no appreciable $\Delta$ FFIR. MEK inhibition with U0126 (b and d) significantly increased $\Delta$ FFIR relative to vehicle treated control strips. Representative traces are shown. Lref: reference length.

sequence, tissues were allowed to relax to baseline in $\mathrm{KH}$ solution containing $500 \mathrm{nM}$ jasplakinolide. Once baseline relaxed tone was achieved, tissues were further incubated in $30 \mu \mathrm{M}$ U0126 (in addition to $500 \mathrm{nM}$ jasplakinolide) for $1 \mathrm{~h}$. A third combined contraction sequence was then performed in the presence of both U0126 and jasplakinolide. These tissues were designated CJU (control-jasplakinolide-jasplakinolide plus U0126) based on the three isotonic/oscillation contraction sequences. Three control groups were also tested, CCC (control-vehicle-vehicle), CJJ (control-jasplakinolide-jasplakinolide) and CCU (control-vehicle-vehicle plus U0126). At the end of the third contraction sequence, but before force fluctuations had stopped, tissues were flash-frozen in liquid nitrogen and transferred to dry-ice-chilled acetone (as above).

\section{Western analysis of target protein phosphorylation}

Proteins from U0126-, jasplakinolide- and vehicle-treated muscles that had been frozen near the end of their respective contraction sequences were extracted as described previously $[16,30]$. All lanes in all gels were loaded with equal concentrations of total protein extract. Denatured proteins were separated by sodium dodecylsulfate-polyacrylamide gel electrophoresis (Invitrogen, Carlsbad, CA, USA), transferred to
Immobilon-P polyvinylidene difluoride (PVDF) membranes and probed for phosphorylated and total ERK1/2, highmolecular-weight caldesmon (h-caldesmon), MLC20, p38 MAPK and Hsp27. Phosphorylated and total proteins were detected on separate PVDF membranes using SuperSignal West Pico chemiluminescent substrate (Thermo, Rockford, IL, USA), and blot intensities (volumes) were calculated using a BioRad densitometer and software (BioRad, Hercules, CA, USA). Ratios of phosphorylated to total protein were expressed relative to data derived for vehicle-treated tissues on the same western blot.

All primary antibodies were raised in rabbits, and were from the following sources: phosphorylated (serine $\left.789\left(\operatorname{Ser}^{789}\right)\right)$ and nonphosphorylated h-caldesmon: L. Adam (Bristol-Myers Squibb, Princeton, NJ, USA); Hsp27 and phosphorylated MLC20: W.T. Gerthoffer (University of South Alabama, Mobile, SL, USA); phosphorylated Hsp27: Assay Designs (Ann Arbor, MI, USA); MLC20: Santa Cruz Biotechnology (Santa Cruz, CA, USA); ERK1/2: M.J. Dunn (Medical College of Wisconsin, Milwaukee, WI, USA); and phosphorylated and nonphosphorylated p38 MAPK and phosphorylated ERK1/2: Cell Signaling (Danvers, MA, USA). 


\section{Data analysis}

All data are expressed as mean \pm SEM. All muscle lengths attained during agonist-elicited isotonic shortening and FFIR were expressed as a percentage of Lref. Differences between the first and second isotonic/force oscillation sequence (i.e. before and after inhibitor or vehicle), or second and third contraction sequences for the jasplakinolide study, were expressed as $\Delta$ FFIR. Results from different groups were compared using ANOVA or a paired or unpaired t-test as appropriate. When ANOVA revealed a difference among means, data were further analysed using the Newman-Keuls test for multiple comparisons. Significant differences were defined when $\mathrm{p}<0.05$.

\section{RESULTS}

\section{MEK inhibition and FFIR in ACh-contracted canine TSM}

For each isotonic/oscillation contraction sequence, FFIR was calculated as the change in contracted muscle strip length that occurred during the 20-min period of oscillation (fig. 1); $\Delta$ FFIR was calculated from these values for each muscle strip. The effects on $\triangle$ FFIR of three concentrations of U0126, 3, 15 and $30 \mu \mathrm{M}$, were compared to that of vehicle alone (fig. 2). U0126 increased the $\triangle$ FFIR of ACh-contracted TSM strips in a concentration-dependent manner $(\mathrm{p}<0.001)$. As in previous studies [16], vehicle-treated tissues demonstrated reproducible FFIR after versus before treatment (i.e. $\Delta \mathrm{FFIR} \sim 0$ ). These data support the hypothesis that MEK regulates $\triangle$ FFIR in canine TSM.

\section{MEK inhibition and isometric force generation or isotonic shortening in ACh-contracted canine TSM}

The effects of 15 and $30 \mu \mathrm{M}$ U0126 on isometric contraction of canine TSM were assessed. No significant shift in the concentration-response relationship with ACh was observed for either concentration of U0126, although strips treated with the higher concentration tended towards reduced force at $1 \times 10^{-4} \mathrm{M} \mathrm{ACh}$

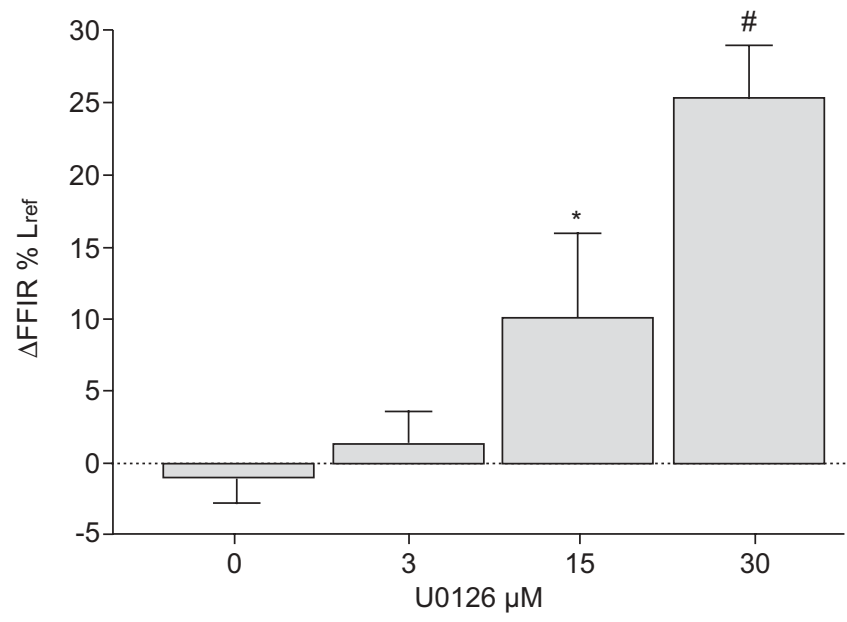

FIGURE 2. Effect of varying concentrations of the mitogen-activated protein kinase kinase (MEK) inhibitor U0126 (1,4-diamino-2,3-dicyano-1,4-bis[2-aminophenylthio]butadiene). The difference $(\Delta)$ in force-fluctuation-induced relengthening (FFIR) was compared to that of acetylcholine-contracted canine tracheal smooth muscle strips that received vehicle alone $(n=14)$. Data are presented as mean \pm SEM. MEK inhibition modulates FFIR in a concentration-dependent manner $(p<0.001$ ( $n=6$ per concentration); ANOVA). *: $p<0.05$ versus no drug treatment; \#: $\mathrm{p}<0.05$ versus 0,3 and $15 \mu \mathrm{M} \cup 0126$ $(p=0.269)$ (fig. 3a). Isotonic shortening after treatment did not differ among tissues treated with $30 \mu \mathrm{M}$ U0126 or vehicle (fig. 3b); as such, this parameter could not account for the differences in $\triangle F F I R$ observed among groups.

\section{MEK inhibition and caldesmon phosphorylation in ACh- contracted canine TSM}

Increasing concentrations of U0126 progressively reduced hcaldesmon phosphorylation ( $\mathrm{p}=0.002$ ) (fig. 4a and $b$ ). As such, $\triangle$ FFIR (which progressively increased with increasing U0126 concentration (fig. 2)) varied inversely with the level of hcaldesmon phosphorylation (fig. 4c). As expected, U0126 treatment substantially reduced ERK1/2 phosphorylation at all concentrations studied (data not shown; $\mathrm{p}<0.001$ ). However, MEK inhibition had no significant effect on the phosphorylation of MLC20, p38 MAPK or Hsp27 (data not shown; $\mathrm{p}>0.25$ for all; ANOVA).
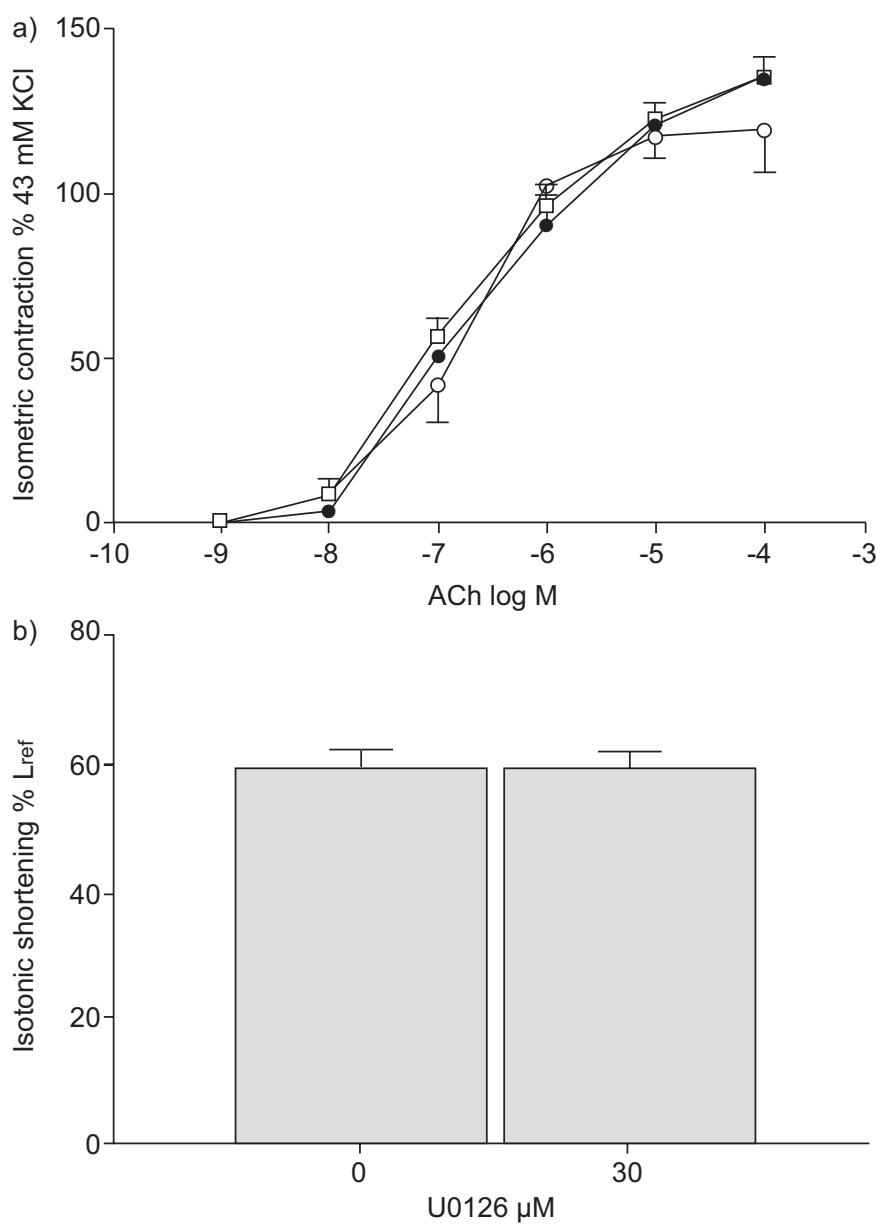

FIGURE 3. Effect of mitogen-activated protein kinase kinase inhibition on isometric responsiveness and isotonic shortening in acetylcholine (ACh)-contracted canine tracheal smooth muscle (TSM) strips. a) U0126 (1,4-diamino-2,3dicyano-1,4-bis[2-aminophenylthio]butadiene) at concentrations of $15(\square ; n=3)$ and $30 \mu \mathrm{M}(O ; n=5)$ had no significant effect on isometric responsiveness compared to control strips $(\bullet ; n=4)(p=0.486 ;$ ANOVA). b) $30 \mu \mathrm{M} \cup 0126(n=6)$ treatment resulted in a substantial increase in change in force-fluctuation-induced relengthening compared to control $(n=14)$, but did not affect isotonic shortening of the TSM strips after treatment $(p=0.951$; unpaired t-test). Data are presented as mean \pm SEM. Lref: reference length. 

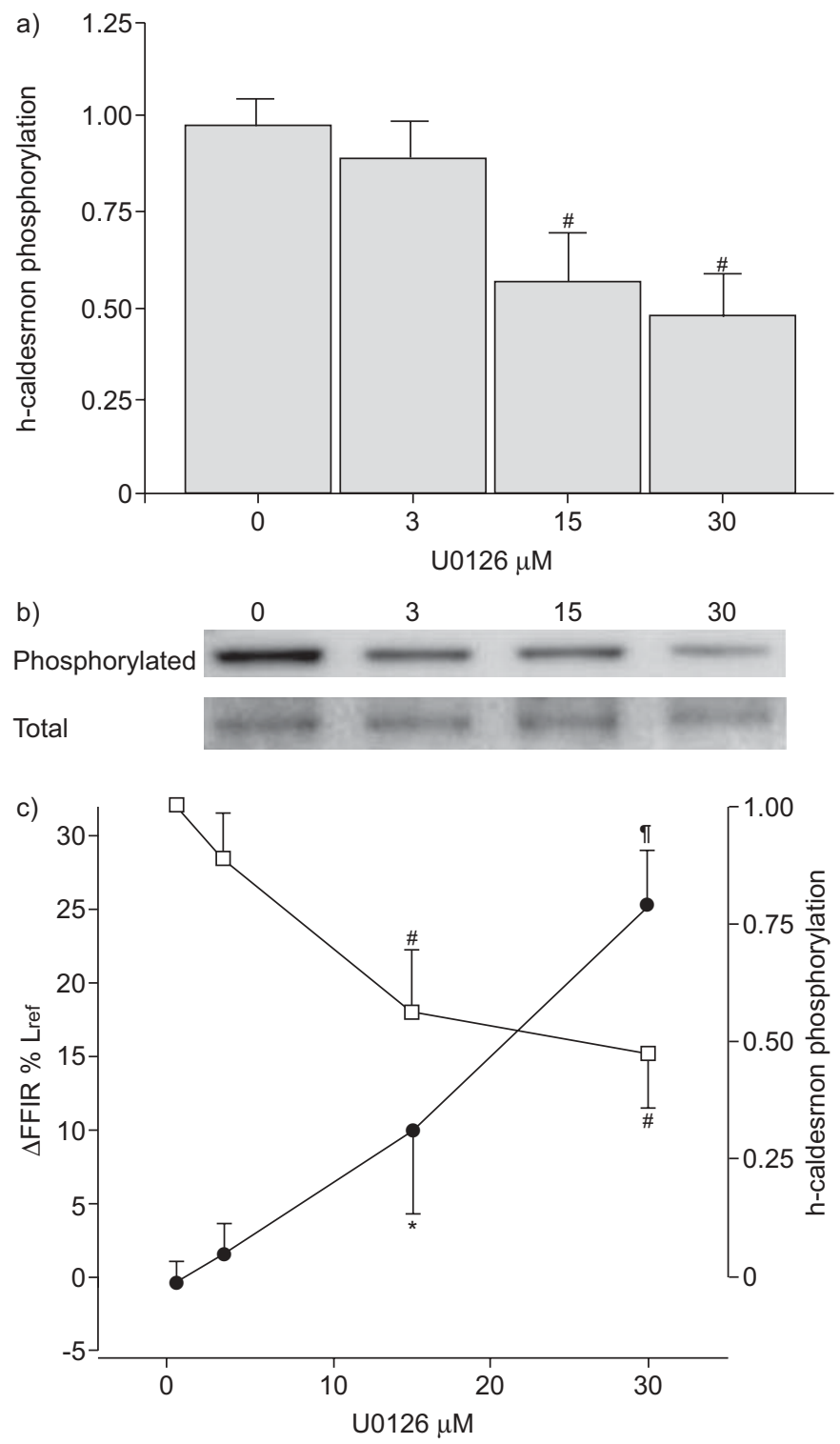

FIGURE 4. Effect of mitogen-activated protein kinase kinase inhibition on caldesmon phosphorylation in canine tracheal smooth muscle (TSM) strips. a) Relative caldesmon phosphorylation levels with $3(n=6), 15(n=5)$ and $30 \mu M(n=6)$ U0126 (1,4-diamino-2,3-dicyano-1,4-bis[2-aminophenylthio]butadiene) in comparison to those in TSM strips that received vehicle alone $(n=10)$; and b) representative corresponding western blot of control- and U0126-treated tissues. Caldesmon demonstrated reduced phosphorylation following treatment with 15 and $30 \mu \mathrm{M}$ U0126 compared to both vehicle-treated and $3-\mu \mathrm{M}$ strips ( $p=0.002$; ANOVA). c) The change in force-fluctuation-induced relengthening $(\triangle \mathrm{FFIR} ; \bullet)$ varied inversely with relative caldesmon phosphorylation $(\square)$ in strips contracted with acetylcholine in the presence of U0126 (data derived from figures 2 and 4a). Data are presented as mean \pm SEM. h-caldesmon: high-molecular-weight caldesmon; Lref: reference length. *: $\mathrm{p}<0.05$ versus no drug treatment; ${ }^{*}$ : versus 0 and $3 \mu \mathrm{M} \cup 0126$; " : versus 0,3 and $15 \mu \mathrm{M} \cup 0126$.

\section{MEK inhibition increases FFIR by a mechanism that does} not involve loss of actin filament integrity

As found previously [16], jasplakinolide alone (CJJ; $\mathrm{n}=3)$ had no effect on FFIR compared to control (CCC; $\mathrm{n}=4)$. Consistent with results from the two-contraction protocol (fig. 1b and d),
$30 \mu \mathrm{M}$ U0126 significantly increased $\Delta$ FFIR in CCU $(n=4)$ tissue strips ( $p=0.004 ;$ ANOVA). CJU $(n=3)$ strips were exposed to jasplakinolide during the second contraction sequence and then jasplakinolide plus U0126 during the third. In these CJU strips, the $\triangle F F I R$ remained equivalent to that observed in CCU tissues $(\mathrm{p}=\mathrm{NS})$. Jasplakinolide did not significantly alter the influence of U0126 on phosphorylation of either ERK1/2 or h-caldesmon (data not shown).

\section{DISCUSSION}

There is a growing body of evidence demonstrating that tidal breathing functionally opposes airway constriction induced by methacholine $[7,10,13]$, and it seems very likely that the FFIR of contracted ASM may account for this anti-bronchoconstrictive effect. Therefore, it is of considerable interest to know what mechanisms determine FFIR and how smooth muscle might be manipulated to accentuate this salutary phenomenon. Previous studies have clearly demonstrated that force fluctuations applied to isotonically contracted ASM result in considerable relengthening despite continued contractile stimulation $[1,4,15,16]$. The present study extends those findings by demonstrating the following. 1) FFIR can be augmented by inhibiting the MEK signalling pathway (figs. 1 and 2). 2) The effect of MEK inhibition on FFIR cannot be accounted for by changes in isometric force generation, isotonic shortening (fig. 3), reduced MLC20 phosphorylation, altered p38 MAPK activation or actin filament dynamics, and so must depend upon some other mechanism. 3) In contrast, the potentiation of FFIR caused by MEK inhibition varies inversely with concomitant inhibition of h-caldesmon phosphorylation (fig. 4c), suggesting that MEK regulates FFIR, at least in part, through its downstream target, h-caldesmon.

Muscarinic receptor activation involves a complex signalling cascade, including activation of MEK and its downstream effectors (fig. 5). Several studies in a variety of species have demonstrated attenuation of contraction in smooth muscles by inhibition of the MEK signalling pathway [19, 31-34]. Notably, D'ANGELO and ADAM [35] demonstrated, in porcine carotid artery, that inhibition of ERK activation using PD 098059 caused attenuation of isometric force, with reductions in both MLC20 and h-caldesmon phosphorylation observed when tissues were activated by endothelin 1. Furthermore, EARLEY et al. [36] showed that knocking down caldesmon expression reduced $\mathrm{KCl}$-stimulated vascular smooth muscle contraction by $62 \%$. Herein, it was sought to assess the effects of MEK inhibition on FFIR in canine TSM without reducing isometric force generation or isotonic shortening. Both 15 and $30 \mu \mathrm{M}$ U0126 clearly had no effect on isotonic shortening (fig. 3b), and, although the higher concentration of U0126 tended to reduce isometric force generation slightly, this agent had absolutely no effect on force generation when used at $15 \mu \mathrm{M}$ (fig. 3a). Nonetheless, substantial and concentration-related augmentation of FFIR was observed. As the primary mechanistic intervention in these studies (i.e. U0126 treatment) directly modified MEK activation, these significant changes indicate that MEK signalling negatively regulates FFIR through a mechanism that does not require alteration of more conventional measures of contraction, i.e. isometric force generation or isotonic shortening. 


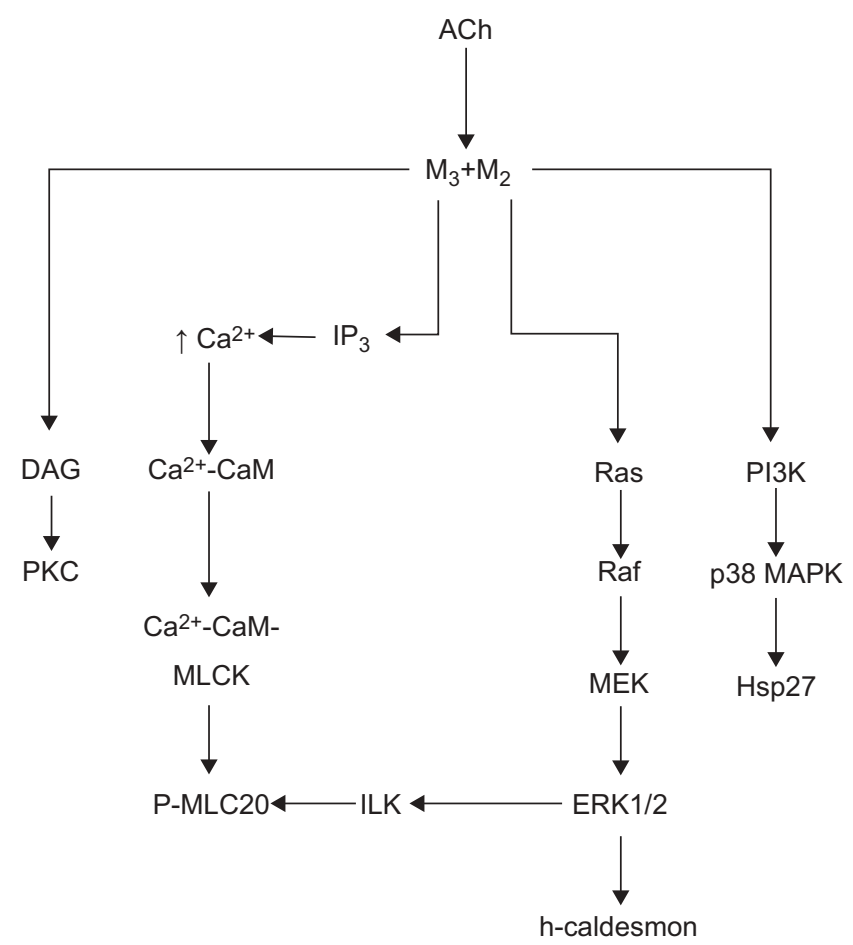

FIGURE 5. Schematic model of the signalling pathways involved in smooth muscle contraction. Acetylcholine (ACh) activates the G-protein-coupled $\mathrm{M}_{2}$ and $\mathrm{M}_{3}$ muscarinic receptors, resulting in the activation of several signalling cascades. $\mathrm{M}_{2}$ activates Raf/mitogen-activated protein kinase (MAPK) kinase (MEK) signalling which, in turn, activates extracellular signal-regulated kinase (ERK) 1/2 and their downstream regulators of contraction, including high-molecular-weight caldesmon (h-caldesmon). Through activation of myosin light chain kinase (MLCK) and ERK1/2, intracellular $\mathrm{Ca}^{2+}$ and integrin-linked kinase (ILK) promote phosphorylation (P) of 20kDa myosin light chain (MLC20). Only a few of the likely effector molecules involved in this pathway are depicted. DAG: diacylglycerol; PKC: protein kinase C; $\mathrm{IP}_{3}$ : inositol 1,4,5-trisphosphate; CaM: calmodulin; PI3K: phosphatidylinositol-3'-kinase; Hsp27: 27-kDa heat shock protein.

Several potential mechanisms by which MEK signalling might have exerted its regulatory influence on FFIR were evaluated. Caldesmon is an actin-associated protein that appears to regulate force in vascular smooth muscle [35]. Work from the laboratory of L.P. Adam has shown that phosphorylation of hcaldesmon parallels isometric force production, and that ERK1/2 are physiologically relevant caldesmon kinases [35]. When caldesmon becomes phosphorylated on $\operatorname{Ser}^{789}$ by active ERK1/2, actomyosin interaction is disinhibited [24-27]. In the present study, U0126 significantly reduced ERK1/2 phosphorylation, and, to a lesser extent, caldesmon phosphorylation, and an inverse relationship was found between $\Delta$ FFIR and level of caldesmon phosphorylation at $\operatorname{Ser}^{789}$ (fig. 4c). Thus the present results suggest a potential role for h-caldesmon phosphorylation, at least on $\mathrm{Ser}^{789}$, in regulating the magnitude of FFIR. Despite inhibition of ERK 1/2 activation by U0126 treatment, partial caldesmon phosphorylation still occurred. Consistent with these findings, others have shown that inhibition of MEK signalling reduces but does not abolish caldesmon phosphorylation [37, 38]. In addition, U0126 at the higher concentrations showed a disproportionate increase in FFIR in relation to the more modest decrease in caldesmon phosphorylation. This suggests that MEK inhibition acts through more than one mechanism to influence FFIR, and perhaps additional inhibition of other purported caldesmon kinases, such as p34 ${ }^{\text {cdc2 }}$ (cyclin-dependent kinase 1) [39], p21activated kinase [40,41], casein kinase II [42] or calmodulin kinase II [43], might enhance FFIR even more dramatically.

Several other potential mechanisms by which MEK inhibition might contribute to augmenting FFIR were also explored. MEK signalling can catalyse the downstream phosphorylation of tropomyosin [44], an actin side-binding protein that stabilises actin filaments. Phosphorylated tropomyosin binds with increased affinity to actin filaments. Therefore, it was hypothesised that MEK signalling might lead to stabilised actin filaments. Since it was previously shown that stabilisation of actin filaments with jasplakinolide blocks latrunculin Binduced increases in FFIR [16], this hypothesis was tested by determining whether jasplakinolide could also block MEKinhibition-induced increases in FFIR. As reported in the results, jasplakinolide pretreatment could not prevent the effect of U0126 on $\triangle$ FFIR. Thus it appears that MEK signalling reduces FFIR through a mechanism that does not involve loss of actin filament integrity. However, it remains conceivable that interaction between actin filaments and the cell membrane at focal adhesions might be involved.

Interestingly, there was no significant change in MLC20 phosphorylation with MEK inhibition. We had previously reasoned that increased actomyosin crossbridge cycling might reduce the net effect of stretch on contracted ASM [4, 8, 15, 45, 46]. In the present study, MLC20 phosphorylation was not affected by U0126 treatment, and tissues treated with or without U0126 shortened similarly (fig. 3b). Since MLC20 phosphorylation is a key determinant of actomyosin crossbridge cycling rate, it seems likely that MEK inhibition had little influence on actomyosin adenosine triphosphatase activity. Thus the MEK pathway regulates FFIR in canine TSM by a mechanism other than through changing MLC20 phosphorylation (or, presumably, actomyosin crossbridge cycling rate). However, it is conceivable that actin-myosin binding per se is impaired with reduced h-caldesmon phosphorylation $[40,47,48]$, which may explain why inhibition of MEK, and thus reduced h-caldesmon phosphorylation, augments FFIR in the present tissues.

Finally, LAKSER et al. [15] had previously shown that p38 MAPK activation inhibits FFIR in contracted bovine TSM. Other studies suggest that crosstalk between the MEK/ERK1/ 2 and p38 MAPK signalling pathways can occur [49-52]. However, since U0126 did not affect the phosphorylation levels of p38 MAPK or Hsp27 in the present studies, MEK inhibition apparently does not exert its influence on FFIR by altering p38 MAPK signalling.

To our knowledge there are no reports of increased MEK activation in asthmatics versus nonasthmatics; however, BURGESS et al. [53] reported an increase in ERK phosphorylation in asthmatic human ASM cells in the presence of low concentrations of fetal bovine serum. Furthermore, DUAN et al. [54] showed that U0126 significantly reduced ovalbumininduced airway hyperresponsiveness to methacholine in their mouse asthma model. Interestingly, ERK phosphorylation was 
also increased in the ovalbumin-challenged mice compared to the naive mice, suggesting a potential role for the MEK/ERK signalling pathway in allergic asthma models.

In conclusion, the present study shows that the MEK signalling pathway regulates the magnitude of FFIR of contracted ASM through a mechanism that, in part, involves h-caldesmon, but does not appear to depend upon changes in MLC20 phosphorylation, p38 MAPK signalling or actin filament integrity. Since FFIR may account, at least in part, for the functional antagonism of bronchoconstriction by tidal breathing, the present results have potential implications for the effects of force oscillations imparted by breathing on airflow obstruction during bronchoconstrictor stimulation. Since FFIR is physiologically regulated (as evidenced by the ability to influence FFIR by manipulation of MEK activity), it seems conceivable that therapeutic intervention to increase FFIR in asthma may be beneficial.

\section{SUPPORT STATEMENT}

This study was supported by National Institutes of Health (Bethesda, MD, USA) grants HL 79368, AI 56352 and HD 043387.

\section{STATEMENT OF INTEREST}

A statement of interest for J. Solway can be found at www.erj. ersjournals.com $/ \mathrm{misc} /$ statements.dtl

\section{REFERENCES}

1 Oliver MN, Fabry B, Marinkovic A, et al. Airway hyperresponsiveness, remodeling, and smooth muscle mass: right answer, wrong reason? Am J Respir Cell Mol Biol 2007; 37: 264-272.

2 Fredberg JJ. Bronchospasm and its biophysical basis in airway smooth muscle. Respir Res 2004; 5: 2.

3 Fredberg JJ, Inouye D, Miller B, et al. Airway smooth muscle, tidal stretches, and dynamically determined contractile states. Am J Respir Crit Care Med 1997; 156: 1752-1759.

4 Fredberg JJ, Inouye DS, Mijailovich SM, et al. Perturbed equilibrium of myosin binding in airway smooth muscle and its implications in bronchospasm. Am J Respir Crit Care Med 1999; 159: 959-967.

5 Gerthoffer WT, Gunst SJ. Invited review: focal adhesion and small heat shock proteins in the regulation of actin remodeling and contractility in smooth muscle. J Appl Physiol 2001; 91: 963-972.

6 Gunst SJ, Fredberg JJ. The first three minutes: smooth muscle contraction, cytoskeletal events, and soft glasses. J Appl Physiol 2003; 95: 413-425.

7 Gunst SJ, Shen X, Ramchandani R, et al. Bronchoprotective and bronchodilatory effects of deep inspiration in rabbits subjected to bronchial challenge. J Appl Physiol 2001; 91: 2511-2516.

8 Latourelle J, Fabry B, Fredberg JJ. Dynamic equilibration of airway smooth muscle contraction during physiological loading. J Appl Physiol 2002; 92: 771-779.

9 Salerno FG, Shinozuka N, Fredberg JJ, et al. Tidal volume amplitude affects the degree of induced bronchoconstriction in dogs. J Appl Physiol 1999; 87: 1674-1677.

10 Shen X, Gunst SJ, Tepper RS. Effect of tidal volume and frequency on airway responsiveness in mechanically ventilated rabbits. J Appl Physiol 1997; 83: 1202-1208.

11 Freedman S, Lane R, Gillett MK, et al. Abolition of methacholine induced bronchoconstriction by the hyperventilation of exercise or volition. Thorax 1988; 43: 631-636.

12 Gump A, Haughney L, Fredberg J. Relaxation of activated airway smooth muscle: relative potency of isoproterenol vs. tidal stretch. J Appl Physiol 2001; 90: 2306-2310.
13 Murphy TM, Ray DW, Alger LE, et al. Ontogeny of dry gas hyperpnea-induced bronchoconstriction in guinea pigs. J Appl Physiol 1994; 76: 1150-1155.

14 Skloot G, Permutt S, Togias A. Airway hyperresponsiveness in asthma: a problem of limited smooth muscle relaxation with inspiration. J Clin Invest 1995; 96: 2393-2403.

15 Lakser OJ, Lindeman RP, Fredberg JJ. Inhibition of the p38 MAP kinase pathway destabilizes smooth muscle length during physiological loading. Am J Physiol Lung Cell Mol Physiol 2002; 282: L1117-L1121.

16 Dowell ML, Lakser OJ, Gerthoffer WT, et al. Latrunculin B increases force fluctuation-induced relengthening of ACh-contracted, isotonically shortened canine tracheal smooth muscle. J Appl Physiol 2005; 98: 489-497.

17 Yamboliev IA, Hedges JC, Mutnick JL, et al. Evidence for modulation of smooth muscle force by the p38 MAP kinase/ HSP27 pathway. Am J Physiol Heart Circ Physiol 2000; 278: H1899-H1907.

18 Cao W, Sohn UD, Bitar KN, et al. MAPK mediates PKC-dependent contraction of cat esophageal and lower esophageal sphincter circular smooth muscle. Am J Physiol Gastrointest Liver Physiol 2003; 285: G86-G95.

19 Gerthoffer WT, Yamboliev IA, Shearer M, et al. Activation of MAP kinases and phosphorylation of caldesmon in canine colonic smooth muscle. J Physiol 1996; 495: 597-609.

20 Kim HR, Hai CM. Mechanisms of mechanical strain memory in airway smooth muscle. Can J Physiol Pharmacol 2005; 83: 811-815.

21 Gerthoffer WT. Signal-transduction pathways that regulate visceral smooth muscle function. III. Coupling of muscarinic receptors to signaling kinases and effector proteins in gastrointestinal smooth muscles. Am J Physiol Gastrointest Liver Physiol 2005; 288: G849-G853.

22 Deng JT, Van Lierop JE, Sutherland C, et al. $\mathrm{Ca}^{2+}$-independent smooth muscle contraction. A novel function for integrin-linked kinase. J Biol Chem 2001; 276: 16365-16373.

23 Harnett KM, Biancani P. Calcium-dependent and calcium-independent contractions in smooth muscles. Am J Med 2003; 115: Suppl. 3A, 24S-30S.

24 Cook AK, Carty $\mathrm{M}$, Singer $\mathrm{CA}$, et al. Coupling of $\mathrm{M}_{2}$ muscarinic receptors to ERK MAP kinases and caldesmon phosphorylation in colonic smooth muscle. Am J Physiol Gastrointest Liver Physiol 2000; 278: G429-G437.

25 D'Angelo G, Graceffa P, Wang CA, et al. Mammal-specific, ERKdependent, caldesmon phosphorylation in smooth muscle. Quantitation using novel anti-phosphopeptide antibodies. J Biol Chem 1999; 274: 30115-30121.

26 Hedges JC, Oxhorn BC, Carty M, et al. Phosphorylation of caldesmon by ERK MAP kinases in smooth muscle. Am J Physiol Cell Physiol 2000; 278: C718-C726.

27 Li Y, Je HD, Malek S, et al. ERK1/2-mediated phosphorylation of myometrial caldesmon during pregnancy and labor. Am J Physiol Regul Integr Comp Physiol 2003; 284: R192-R199.

28 Mitchell RW, Antonissen LA, Kepron W, et al. Effect of atropine on the hyperresponsiveness of ragweed-sensitized canine tracheal smooth muscle. J Pharmacol Exp Ther 1986; 236: 803-809.

29 Lakser OJ, Dowell ML, Hoyte FL, et al. Steroids augment relengthening of contracted airway smooth muscle: potential additional mechanism of benefit in asthma. Eur Respir J 2008; 32: 1224-1230.

30 Mitchell RW, Halayko AJ, Kahraman S, et al. Selective restoration of calcium coupling to muscarinic $\mathrm{M}_{3}$ receptors in contractile cultured airway myocytes. Am J Physiol Lung Cell Mol Physiol 2000; 278: L1091-L1100.

31 Lagaud GJ, Lam E, Lui A, et al. Nonspecific inhibition of myogenic tone by PD98059, a MEK1 inhibitor, in rat middle cerebral arteries. Biochem Biophys Res Commun 1999; 257: 523-527. 
32 Pearce WJ, Williams JM, Chang MM, et al. ERK inhibition attenuates 5-HT-induced contractions in fetal and adult ovine carotid arteries. Arch Physiol Biochem 2003; 111: 36-44.

33 Xiao D, Pearce WJ, Longo LD, et al. ERK-mediated uterine artery contraction: role of thick and thin filament regulatory pathways. Am J Physiol Heart Circ Physiol 2004; 286: H1615-H1622.

34 Xiao D, Zhang L. ERK MAP kinases regulate smooth muscle contraction in ovine uterine artery: effect of pregnancy. Am J Physiol Heart Circ Physiol 2002; 282: H292-H300.

35 D'Angelo G, Adam LP. Inhibition of ERK attenuates force development by lowering myosin light chain phosphorylation. Am J Physiol Heart Circ Physiol 2002; 282: H602-H610.

36 Earley JJ, Su X, Moreland RS. Caldesmon inhibits active crossbridges in unstimulated vascular smooth muscle: an antisense oligodeoxynucleotide approach. Circ Res 1998; 83: 661-667.

37 Gorenne I, Su X, Moreland RS. Caldesmon phosphorylation is catalyzed by two kinases in permeabilized and intact vascular smooth muscle. J Cell Physiol 2004; 198: 461-469.

38 Krymsky MA, Chibalina MV, Shirinsky VP, et al. Evidence against the regulation of caldesmon inhibitory activity by $\mathrm{p} 42 / \mathrm{p} 44^{\text {erk }}$ mitogen-activated protein kinase in vitro and demonstration of another caldesmon kinase in intact gizzard smooth muscle. FEBS Lett 1999; 452: 254-258.

39 Mak AS, Carpenter M, Smillie LB, et al. Phosphorylation of caldesmon by $\mathrm{p} 34^{\mathrm{cdc} 2}$ kinase. Identification of phosphorylation sites. J Biol Chem 1991; 266: 19971-19975.

40 Foster DB, Shen LH, Kelly J, et al. Phosphorylation of caldesmon by $\mathrm{p} 21$-activated kinase. Implications for the $\mathrm{Ca}^{2+}$ sensitivity of smooth muscle contraction. J Biol Chem 2000; 275: 1959-1965.

41 McFawn PK, Shen L, Vincent SG, et al. Calcium-independent contraction and sensitization of airway smooth muscle by p21activated protein kinase. Am J Physiol Lung Cell Mol Physiol 2003; 284: L863-L870.

42 Vorotnikov AV, Gusev NB, Hua S, et al. Identification of casein kinase II as a major endogeneous caldesmon kinase in sheep aorta smooth muscle. FEBS Lett 1993; 334: 18-22.

43 Ikebe M, Reardon S. Phosphorylation of smooth myosin light chain kinase by smooth muscle $\mathrm{Ca}^{2+} /$ calmodulin-dependent multifunctional protein kinase. J Biol Chem 1990; 265: 8975-8978.
44 Houle F, Rousseau S, Morrice N, et al. Extracellular signalregulated kinase mediates phosphorylation of tropomyosin-1 to promote cytoskeleton remodeling in response to oxidative stress: impact on membrane blebbing. Mol Biol Cell 2003; 14 1418-1432.

45 Dulin NO, Fernandes DJ, Dowell $\mathrm{M}$, et al. What evidence implicates airway smooth muscle in the cause of BHR? Clin Rev Allergy Immunol 2003; 24: 73-84.

46 Solway J, Bellam S, Dowell M, et al. Actin dynamics. A potential integrator of smooth muscle (dys-)function and contractile apparatus gene expression in asthma. Parker B. Francis lecture. Chest 2003; 123: 392S-398S.

47 Huang $\mathrm{R}$, Li L, Guo $\mathrm{H}$, et al. Caldesmon binding to actin is regulated by calmodulin and phosphorylation via different mechanisms. Biochemistry 2003; 42: 2513-2523.

48 Kulikova N, Avrova SV, Borovikov YS. Caldesmon inhibits the rotation of smooth actin subdomain-1 and alters its mobility during the ATP hydrolysis cycle. Biochem Biophys Res Commun 2009; 390: 125-129.

49 Houliston RA, Pearson JD, Wheeler-Jones CP. Agonist-specific cross talk between ERKs and $\mathrm{p} 38^{\text {mapk }}$ regulates $\mathrm{PGI}_{2}$ synthesis in endothelium. Am J Physiol Cell Physiol 2001; 281: C1266-C1276.

50 Kurosawa M, Numazawa S, Tani Y, et al. ERK signaling mediates the induction of inflammatory cytokines by bufalin in human monocytic cells. Am J Physiol Cell Physiol 2000; 278: C500-508.

51 Liu Q, Hofmann PA. Protein phosphatase 2A-mediated cross-talk between p38 MAPK and ERK in apoptosis of cardiac myocytes. Am J Physiol Heart Circ Physiol 2004; 286: H2204-H2212.

52 Xiao YQ, Malcolm K, Worthen GS, et al. Cross-talk between ERK and p38 MAPK mediates selective suppression of pro-inflammatory cytokines by transforming growth factor- $\beta$. J Biol Chem 2002; 277: 14884-14893.

53 Burgess JK, Lee JH, Ge Q, et al. Dual ERK and phosphatidylinositol 3-kinase pathways control airway smooth muscle proliferation: differences in asthma. J Cell Physiol 2008; 216: 673-679.

54 Duan $\mathrm{W}$, Chan JH, Wong $\mathrm{CH}$, et al. Anti-inflammatory effects of mitogen-activated protein kinase kinase inhibitor U0126 in an asthma mouse model. J Immunol 2004; 172: 7053-7059. 